\title{
Modeling of sand-water slurry flow through horizontal pipe using CFD
}

\author{
Manoj Kumar Gopaliya ${ }^{1}$, D.R. Kaushal ${ }^{2 *}$ \\ ${ }^{1}$ Department of Mechanical Engineering, The NorthCap University (Formerly ITM University), Gurgaon, Sector-23 A, Gurgaon-122017, \\ India. Tel.: +91-8860759682 (M), +91-124-4195263 (O). Fax: +91-124-2367488 \\ 2 Department of Civil Engineering, Indian Institute of Technology, Delhi, Hauz Khas, New Delhi-110016, India. \\ * Corresponding author. Tel.: +91-9818280867 (M) \& +91-11-26591216 (O). Fax: +91-11-2658 1117. E-mail: kaushal@civil.iitd.ac.in
}

\begin{abstract}
The paper presents three-dimensional CFD analysis of two-phase (sand-water) slurry flows through $263 \mathrm{~mm}$ diameter pipe in horizontal orientation for mixture velocity range of 3.5-4.7 m/s and efflux concentration range of 9.95$34 \%$ with three particle sizes viz. $0.165 \mathrm{~mm}, 0.29 \mathrm{~mm}$ and $0.55 \mathrm{~mm}$ with density $2650 \mathrm{~kg} / \mathrm{m}^{3}$. RNG $k-\varepsilon$ turbulence closure equations with Eulerian multi-phase model is used to simulate various slurry flows. The simulated values of local solid concentration are compared with the experimental data and are found to be in good agreement for all particle sizes. Effects of particle size on various slurry flow parameters such as pressure drop, solid phase velocity distribution, friction factor, granular pressure, turbulent viscosity, turbulent kinetic energy and its dissipation have been analyzed.
\end{abstract}

Keywords: CFD; Slurry flow; Particle size; Solid concentration; Pipeline.

\section{INTRODUCTION}

Solid transport through slurry flow is becoming increasingly popular due to ever increasing emphasis of society on reduction in environmental pollution. The features like reduction in traffic, air pollution, noise, accidents along with lesser ecological disturbance and saving on energy consumption is the reason for this growing popularity of slurry transportation systems. Presently, mineral, chemical and power industries are predominantly using this mode of transportation. Here solids to be transported are pumped through pipelines in the form of slurries. It is therefore needed to determine the most efficient and economical process of pumping these solids in carrier liquid. This requires careful analysis of different factors effecting the performance and cost of whole process including environmental regulations of the land. This itself requires more basic and fundamental understanding of different aspects of the material behavior especially variance in its properties during slurry flows.

Researches since the start of third decade of $20^{\text {th }}$ century are aiming towards developing general solutions based on limited sets of experimental data for solid concentration profiles, pressure drop and deposition velocity which are primarily required for better understanding of whole slurry flow process. Aude et al. (1974), Aude et al. (1975) and Seshadri $(1979,1982)$ and Seshadri et al. (1982) highlighted several attractive features responsible for the acceptance of long distance slurry pipeline transportation system as a viable mode of transportation. Among the initial researches, O'Brien (1933) and Rouse (1937) predicted the concentration distribution using diffusion model for open channel flows having very low volumetric solid concentration. From then, many researches took place aiming at predicting concentration distribution for slurry flows. A few notable of them are Shook and Daniel (1965), Shook et al. (1968), Karabelas (1977), Seshadri et al. (1982), Roco and Shook (1983), Roco and Shook (1984), Gillies et al. (1991), Gillies and Shook (1994), Gillies et al. (1999), Gillies and Shook (2000), Kaushal and Tomita (2002), Kaushal and Tomita (2003) and Kaushal et al.(2005). Apart from this, several studies for predicting pressure drop over the length also took place for slurry flows. A few notable of them are Wasp et al.(1970), Doron et al.(1987), Gillies et al. (1991), Sundqvist et al. (1996),
Mishra et al. (1998), Ghanta and Purohit (1999), Wilson et al. (2002), Kaushal and Tomita (2002), Kaushal and Tomita (2003) and Kaushal et al. (2005).

With ever increasing computational efficiency, the cost-tocomputing expense ratio is decreasing. This has helped Computational Fluid Dynamics (CFD) in becoming one of the most attractive tool for analyzing slurry flows through pipes and as well as in open channels. In spite of having its limitations, CFD is very productive in designing new processes in slurry flows and also in predicting its behavior due to variations in the different process parameters. It helps researcher in conducting detailed parametric studies with greater ease and at very low cost by providing detailed and exhaustive information about the local variations of flow parameters within the flow domain. The same is otherwise quite tedious and many times impossible during experiments.

Krampa-Morlu et al. (2004) studied the flow in a vertical pipeline using per phase $k-\varepsilon$ turbulence model for slurry flows with courser particles. They investigated the effect of particle size, efflux concentration and viscosity on various process parameters. Ling et al. (2003) obtained the numerical solution for sand-water slurry flow in a straight horizontal pipe for a range of mean solid concentrations using algebraic slip mixture $(A S M)$ model. They found simulation data to be in good agreement with experimental data for mixture velocities higher than the deposition velocity. Lin and Ebadian (2008) investigated sand-water slurry flows in the entrance region of horizontal pipeline using CFD with $A S M$ model and showed the progress of various flow parameters in the entrance region of horizontal pipeline. The numerical investigation of slurry flows through horizontal pipes of different diameters by Ekambara et al. (2009) obtained results which were in good agreement with laboratory results for particles smaller than $270 \mu \mathrm{m}$ especially in the central core region. Chen et al. (2009) did similar numerical simulations but for relatively smaller pipe cross-sections. The results here were also found to be in good agreement with laboratory results. Kaushal et al. (2012) performed modeling of slurry pipeline flow shaving fine particles at higher solid concentrations using CFD. They investigated the flows using Mixture and Eulerian two-phase models separately and concluded recommending Eulerian model for these flows as the model predicted pressure drop (per metre length) and solid concentra- 
tion profiles quite accurately for all the cases. Antaya et al. (2012) investigated CFD modeling of concentrated slurry pipeline flows and presented the effect of various turbulence models, wall boundary conditions and type of inter-phase forces on the outcome of numerical study. Wang et al. (2013a) investigated various flow parameters of heterogeneous ice slurry at different operating conditions using CFD. They adopted Mixture multiphase model based on different rheological behavior to characterize the flow. Wang et al. (2013b) investigated detailed flow information of ice slurry using CFD. Eulerian multiphase model based on the kinetic theory of granular flow adopted for analysis where ice slurry flow, ice particle concentration and pressure drop in horizontal, vertical and $90^{\circ}$ elbow pipes were obtained. Wu et al. (2015) investigated, both experimentally and numerically using CFD, the slurry flow of cemented coal gangue-fly ash $(C G F)$ mixtures with emphasize on pressure drop for different mix proportions. Effects of slurry flow rate and pumping pressure along with the effect of pipe size and flow velocity on pressure drop were demonstrated. Ma et al. (2015) investigated erodent particle trajectories in slurry flow using CFD. Discrete phase model (DPM) and volume of fluid method $(V O F)$ is adopted to calculate movement of erodent particles and interface between liquid phase and gas phase respectively. Gopaliya and Kaushal (2015) presented the effect of particle sizes on various flow parameters of sand-water slurry through $53.2 \mathrm{~mm}$ pipe using CFD. This paper predominantly presented the effect of particle size on various slurry flow parameters using qualitative results.

The present work presents CFD modeling of two-phase (sand-water) slurry flows through $263 \mathrm{~mm}$ diameter horizontal pipe for mixture velocity range of $3.5-4.7 \mathrm{~m} / \mathrm{s}$ and efflux concentration range of $9.95-34 \%$ with three particle sizes viz. $0.165 \mathrm{~mm}, 0.29 \mathrm{~mm}$ and $0.55 \mathrm{~mm}$. Eulerian multi-phase model with RNG k- $\varepsilon$ turbulence closure equations is adopted to simulated the mono-dispersed sand particles having density $2650 \mathrm{~kg} / \mathrm{m}^{3}$. Fluent 6.3 .26 is used for CFD analysis of slurry flows. The simulation results are compared with available experimental values of solid concentration distribution (Roco and Shook (1983) and Gillies and Shook (1994)). Detailed quantitative analysis of effect of particle size on various slurry flow parameters has been presented in this paper including the friction factor growth along the pipe length and turbulence aspects of slurry flows, addressed very first time.

\section{MATHEMATICAL MODEL}

The selection of appropriate multiphase model is foremost important in the CFD analysis of slurry flows and it depends mainly on the range of volume fraction $(\alpha)$ of solid phase under consideration. In the view of high range of solid volume fractions under consideration, Granular version of Eulerian model has been adopted for simulations during present study. Granular version has the capability of capturing effect of friction and collusions between particles which is very much important in higher concentration slurry flows with different particle sizes.

\section{Eulerian model}

Two-phase Eulerian model adopted in present research assumes that the slurry flow consists of solid phase " $s$ " and fluid phase " $f$ ". These phases are assumed to be separated, yet forming interpenetrating continua such that $\alpha_{s}+\alpha_{f}=100 \%$. Here $\alpha_{s}$ and $\alpha_{f}$ are the volumetric concentrations of solid and fluid phases respectively. Continuity and momentum equations are individually satisfied by each phase. Coupling of equations of these two phases is achieved using pressure and inter-phasial exchange coefficients. The forces acting on a single particle in the slurry are summarized below:

i. $\quad$ Static pressure gradient, $\nabla P$.

ii. Solid pressure gradient or the inertial force due to particle interactions, $\nabla P S$ which is defined using volumetric concentration, density \& granular temperature of solid phase along with radial distribution of particles and loss of kinetic energy due to their collisions, represented by restitution coefficient.

iii. Drag force caused by the velocity difference between two phases, $K_{s f}\left(\vec{v}_{s}-\vec{v}_{f}\right)$ where $K_{s f}$ is the inter-phase drag coefficient and $\overrightarrow{v_{s}} \& \overrightarrow{v_{f}}$ are velocity of solid and fluid phase respectively. Inter-phase drag coefficient $K_{s f}$ is derived using volumetric concentrations of solid and liquid phases, liquid density, particle size, drag coefficient, terminal velocity of solid phase and velocity of solid and liquid phases.

iv. Viscous forces, $\nabla \cdot \overline{\overline{\tau_{f}}}$ where $\overline{\overline{\tau_{f}}}$ is the stress tensor for fluid.

v. Body forces, $\rho_{f} \vec{g}$, where, $\rho_{f}$ is the density and $g$ is acceleration due to gravity.

vi. Virtual mass force, $C_{v m} \alpha_{s} \rho_{f}\left(\overrightarrow{v_{f}} \cdot \nabla \overrightarrow{v_{f}}-\overrightarrow{v_{s}} \cdot \nabla \overrightarrow{v_{s}}\right)$ where $C_{v m}$ is the coefficient of virtual mass force. Since in most of the multiphase applications, the effective particle radius is very small as compared to velocity scale, virtual mass effects can be observed predominantly only at relatively high efflux concentrations. It is therefore a value of 0.5 (default value) is adopted for $C_{v m}$ in the present study which in absence of spatial velocity gradients reduces the acceleration term in momentum equation to classically accepted virtual mass force.

vii. Lift force, $C_{L} \alpha_{s} \rho_{f}\left(\overrightarrow{v_{s}}-\overrightarrow{v_{f}}\right) \times\left(\nabla \times \overrightarrow{v_{f}}\right)$ where $C_{L}$ is the lift coefficient taken as 0.5 (Default value).

Governing Equations

Continuity Equation

$$
\nabla .\left(\alpha_{t} \rho_{t} \vec{v}_{f} \vec{v}_{f}\right)=0
$$

where, $t$ is either $s$ or $f$.

\section{$\underline{\text { Momentum Equations }}$}

For fluid phase:

$$
\begin{aligned}
& \nabla .\left(\alpha_{f} \rho_{f} \vec{v}_{f} \vec{v}_{f}\right)=-\alpha_{f} \nabla P+\nabla \cdot \overline{\overline{\tau_{f}}}+\alpha_{f} \rho_{f} \vec{g}+K_{s f}\left(\vec{v}_{s}-\vec{v}_{f}\right)+ \\
& C_{v m} \alpha_{f} \rho_{f}\left(\vec{v}_{s} . \nabla \vec{v}_{s}-\vec{v}_{f} \cdot \nabla \vec{v}_{f}\right)+C_{L} \alpha_{s} \rho_{f}\left(\vec{v}_{f}-\vec{v}_{s}\right) \times\left(\nabla \times \vec{v}_{f}\right)
\end{aligned}
$$

For solid phase:

$$
\begin{aligned}
& \nabla .\left(\alpha_{s} \rho_{s} \vec{v}_{s} \vec{v}_{s}\right)=-\alpha_{s} \nabla P-\nabla P_{s}+\nabla \cdot \overline{\tau_{s}}+\alpha_{s} \rho_{f} \vec{g}+K_{f s}\left(\vec{v}_{f}-\vec{v}_{s}\right) \\
& +C_{v m} \alpha_{s} \rho_{f}\left(\vec{v}_{f} \cdot \nabla \vec{v}_{f}-\vec{v}_{s} \cdot \nabla \vec{v}_{s}\right)+C_{L} \alpha_{s} \rho_{f}\left(\vec{v}_{s}-\vec{v}_{f}\right) \times\left(\nabla \times \vec{v}_{f}\right) \\
& \text { where } \overline{\overline{\tau_{s}}} \text { and } \overline{\overline{\tau_{f}}} \text { are the stress tensors for solid and fluid, }
\end{aligned}
$$


respectively and are expressed as follows:

$$
\begin{aligned}
& \overline{\overline{\tau_{s}}}=\alpha_{s} \mu_{s}\left(\nabla \vec{v}_{s}+\nabla \vec{v}_{s}^{t r}\right)+\alpha_{s}\left(\lambda_{s}-\frac{2}{3} \mu_{s}\right) \nabla \cdot \vec{v}_{s} \overline{\bar{I}} \\
& \text { and } \overline{\overline{\tau_{f}}}=\alpha_{f} \mu_{f}\left(\nabla \vec{v}_{f}+\nabla \vec{v}_{f}^{t r}\right)
\end{aligned}
$$

Here superscript ' $t r$ ' over velocity vector indicates transpose and $\overline{\bar{I}}$ indicates identity tensor. $\lambda_{\mathrm{s}}$ represents bulk viscosity of the solid represented as below:

$\lambda_{s}=\frac{4}{3} \alpha_{s} \rho_{s} d_{s} g_{o, s s}\left(1+e_{s s}\right)\left(\frac{\Theta_{s}}{\pi}\right)^{\frac{1}{2}}$

$d_{s}$ is the particle diameter. $g_{o, s s}$ is the radial distribution function, which is interpreted as the probability of particle touching another particle:

$g_{o, s s}=\left[1-\left(\frac{\alpha_{s}}{\alpha_{s, \max }}\right)^{\frac{1}{3}}\right]^{-1}$

Here, $\alpha_{\mathrm{s}, \max }$ is the static settled concentration, $\Theta_{s}$ is the granular temperature, which is proportional to the kinetic energy of the fluctuating particle motion, $\mathrm{e}_{\mathrm{ss}}$ is the restitution coefficient. Restitution coefficient $\left(\mathrm{e}_{\mathrm{ss}}\right)$ indicates how much kinetic energy remains after collision of particles. Its value ranges from zero to unity. Unity indicates no or very little loss of kinetic energy during collision whereas zero indicates loss of total or very large fraction of kinetic energy during collisions. During present study, a value of 0.9 is adopted for restitution coefficient.

$\mu_{\mathrm{f}}$ is the shear viscosity of fluid and $\mu_{\mathrm{s}}$ represents shear viscosity of solids defined as:

$\mu_{s}=\mu_{s, c o l}+\mu_{s, k i n}+\mu_{s, f r}$

where $\mu_{s, c o l}, \mu_{s, k i n}$ and $\mu_{s, f r}$ are collisional, kinetic and frictional viscosity which are modeled using strain rate, solid pressure, and friction angle, inter-phasial momentum exchange coefficient, drag co-efficient, relative Reynolds number and terminal velocity.

$K_{s f}$ is the inter-phasial momentum exchange coefficient given by:

$K_{s f}=K_{f s}=\frac{3}{4} \frac{\alpha_{s} \alpha_{f} \rho_{f}}{V_{r, s}^{2} d_{s}} C_{D}\left(\frac{\mathrm{Re}_{s}}{V_{r, s}}\right)\left|\vec{v}_{s}-\vec{v}_{f}\right|$

$C_{D}$ is the drag coefficient given by:

$C_{D}=\left[0.63+4.8\left(\frac{\mathrm{Re}_{s}}{V_{r, s}}\right)^{\frac{-1}{2}}\right]^{2}$

$R e_{s}$ is the relative Reynolds number between phases ' $f$ ' and ' $\mathrm{s}$ ' and is given by:

$\operatorname{Re}_{s}=\frac{\rho_{f} d_{s}\left|\vec{v}_{s}-\vec{v}_{f}\right|}{\mu_{f}}$

$V_{r, s}$ is the terminal velocity correlation for solid phase given by:

$V_{r, s}=0.5\left(A-0.06 \mathrm{Re}_{S}+\sqrt{\left(0.06 \mathrm{Re}_{s}\right)^{2}+0.12 \mathrm{Re}_{S}(2 B-A)+A^{2}}\right.$ with $A=\alpha_{f}^{4.14} ; B=0.8 \alpha_{f}^{1.28}$ for $\alpha_{f} \leq 0.85$

and $A=\alpha_{f}^{4.14} ; B=\alpha_{f}^{2.65}$ for $\alpha_{f}>0.85$

\section{Turbulence closure for the fluid phase}

Turbulent quantities for the fluid phase are predicted using RNG $k-\varepsilon$ model (Launder and Spalding, 1974) supplemented by additional terms accounting interfacial turbulent momentum transfer.

The Reynolds stress tensor for the fluid phase " $f$ " is:

$\overline{\overline{\tau_{t, f}}}=-\frac{2}{3}\left(\rho_{f} k_{f}+\mu_{t, f} \nabla \vec{v}_{f}\right) \overline{\bar{I}}+\mu_{t, f}\left(\nabla \vec{v}_{f}+\nabla \vec{v}_{f}^{t r}\right)$

Here $\mu_{t, f}$ is the turbulent viscosity. RNG theory provides an analytically-derived differential correlation for turbulent viscosity that accounts for low Reynolds number effects. This correlation in the high Reynolds number limit (as the cases in present study) converts to:

$\mu_{t, f}=\rho_{f} C_{\mu} \frac{k_{f}^{2}}{\varepsilon_{f}}$ with $C_{\mu}=0.09$

The predictions of turbulent kinetic energy $k_{f}$ and its rate of dissipation $\varepsilon_{f}$ in RNG $k-\varepsilon$ model are similar to Standard $k-\varepsilon$ model, described widely in published researches on CFD.

The main difference between the RNG and standard $k-\varepsilon$ models lies in the additional term in the $\varepsilon$ equation given by:

$R_{\varepsilon}=\frac{C_{\mu} \rho \eta^{3}\left(1-\eta / \eta_{o}\right)}{1+\beta \eta^{3}} \frac{\varepsilon^{2}}{k}$

where $\eta \equiv S k / \varepsilon, \eta_{o}=4.38, \beta=0.012$. The constant parameters used in different equations are taken as: $C_{1 \varepsilon}=1.42, C_{2 \varepsilon}=$ $1.68, C_{3 \varepsilon}=1.2, \sigma_{k}=1.0$ and $\sigma_{\varepsilon}=1.3$.

\section{Turbulence in the solid phase}

Turbulence in solid phase is predicted using Tchen's theory (Lun et al., 1984) of the dispersion of discrete particle in homogeneous and steady turbulent flow. In this theory, dispersion coefficients, correlation functions and turbulent kinetic energy of the solid phase are modeled using the characteristics of continuous turbulent motions of fluid phase which itself is based on time scale and characteristic time. The time scale considering inertial effects acting on the particle is given as follows:

$\tau_{F, s f}=\alpha_{s} \rho_{f} K_{s f}^{-1}\left(\frac{\rho_{s}}{\rho_{f}}+C_{v m}\right)$

The characteristic time of correlated turbulent motion or eddy particle interaction time is given by:

$\tau_{t, s f}=\tau_{t, f}\left[1+C_{\beta} \xi^{2}\right]^{\frac{-1}{2}}$

$\xi=\frac{\left|\vec{V}_{r}\right|}{\sqrt{\frac{2}{3} k_{f}}}$ 

by:

The characteristic time of energetic turbulent eddies is given

$\tau_{t, f}=\frac{3}{2} C_{\mu} \frac{k_{f}}{\varepsilon_{f}}$

$\left|\vec{V}_{r}\right|$ is the average value of the local relative velocity between particle and surrounding fluid defined as the difference in slip and drift velocity $\left(\vec{V}_{r}=\vec{v}_{s f}-\vec{v}_{d r}\right)$.

\section{Transport equation for granular temperature $(\Theta \mathrm{s})$}

The granular temperature for solid phase is described using the kinetic energy (Gidaspow et al., 1992) of random motion of solid particles. The transport equation derived from this theory is given by:

$$
\frac{3}{2} \nabla \cdot\left(\rho_{s} \alpha_{s} \vec{v}_{s} \Theta_{s}\right)=\left(-P_{s} \overline{\bar{I}}+\overline{\overline{\tau_{s}}}\right): \nabla \vec{v}_{s}+\nabla \cdot\left(k_{\Theta s} \nabla \Theta_{s}\right)-\gamma_{\Theta s}+\varphi_{f s}
$$

where the term $\left(-P_{s} \overline{\bar{I}}+\overline{\overline{\tau_{s}}}\right): \nabla \vec{v}_{s}$ defines the generation of energy by the solid stress tensor. And, $k_{\Theta s}$ is the diffusion coefficient modeled as:

$k_{\Theta s}=\frac{15 d_{s} \rho_{s} \alpha_{s} \sqrt{\Theta_{s} \pi}}{4(41-33 \eta)}\left[\begin{array}{l}1+\frac{12}{5} \eta^{2}(4 \eta-3) \alpha_{s} g_{o, s s} \\ +\frac{16}{15 \pi}(41-33 \eta) \eta \alpha_{s} g_{o, s s}\end{array}\right]$

$k_{\Theta s} \nabla \Theta_{s}$ as a whole represents the diffusive flux of granular energy; and

$\eta=\frac{1}{2}\left(1+e_{s s}\right)$

$\gamma_{\Theta s}$ is the collisional dissipation energy representing the energy dissipation rate within the solid phase due to collision between particles. It is modeled as:

$\gamma_{\Theta s}=\frac{12\left(1-e_{s s}^{2}\right) g_{o, s s}}{d_{s} \sqrt{\pi}} \rho_{s} \alpha_{s}^{2} \Theta_{s}^{\frac{3}{2}}$

$\varphi_{f_{S}}$ is the transfer of the kinetic energy of random fluctuation in particle velocity from solid phase ' $\mathrm{s}$ ' to the fluid phase ' $\mathrm{f}$ '. It is modeled as:

$\varphi_{f s}=-3 K_{f s} \Theta_{s}$

For further details of modeling/derivation of variables used in the equations listed in above paragraphs, refer Gopaliya and Kaushal (2015).

\section{Wall functions}

The region very near to the walls needs special attention due to the presence of very high gradients of quantities involve in the analysis. This is achieved using a very fine boundary layer meshing along with the use of standard wall functions option available with RNG $k-\varepsilon$ model. These wall functions are a col- lection of semi-empirical formulas and functions. This helps in achieving lower calculation efforts with better results near the wall.

\section{SIMULATION METHODOLOGY Geometry and mesh generation}

The computational grids for $263 \mathrm{~mm}$ internal diameter $(D)$ horizontal pipe with $50 \mathrm{D}$ length (to ensure fully developed flow) is generated using GAMBIT 2.4.6. Cartesian coordinate system having origin at centre of the pipe inlet cross-section is adopted for geometry generation. The grid contains hexahedral volume cells in sufficient numbers finalized through due mesh independency process. During present study, different computational grids with progressive increase in volume cells at the rate of $50 \%$ have been investigated. The grid showing improved results than grids with lesser number elements along with results comparable to grids having higher number of elements is finalized for simulation. A 15 layer boundary region with a growth rate of $20 \%$ is created near the boundary. This is done to improve the performance of the wall functions adopted to capture the gradient near the walls and also to fulfill the convergence requirement of $y^{+}=30$ where $y^{+}$is the dimensionless wall distance of cells next to the wall. The numerical grid at pipe outlet is shown in Figure 1.

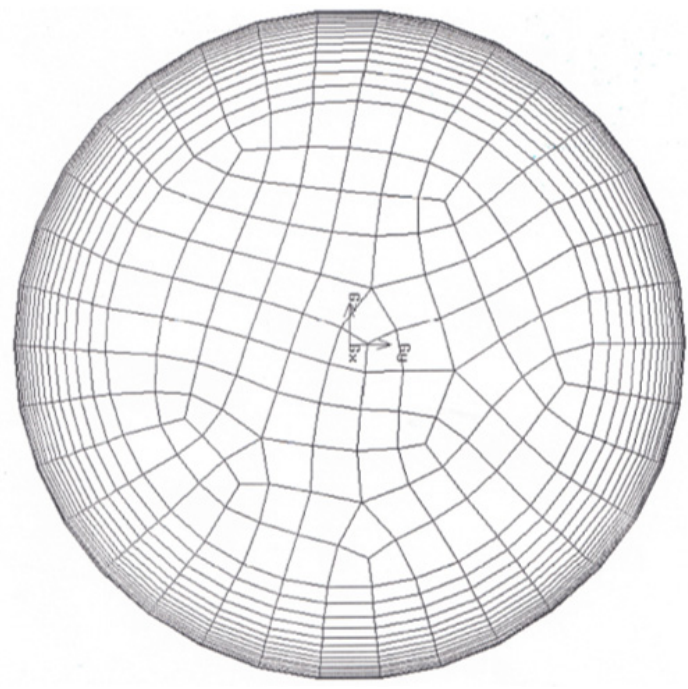

Fig. 1. Numerical grid.

\section{Boundary conditions}

There are three boundaries available in the given flow domain namely the inlet boundary, the wall boundary and the outlet boundary. Flat velocity and volume fraction of both the phases, liquid and solid, were notified at the inlet boundary, i.e., $V_{m}=v_{s}=v_{f}, \alpha_{s}$ as $=C_{v f}$ and $\alpha_{f}=1-C_{v f}$. Here $V_{m}$ is the mean flow velocity, measured in laboratory by Roco and Shook (1983) and Gillies and Shook (1994); $C_{v f}$ is the efflux concentration in the slurry pipe and is expressed as:

$$
C_{v f}=\frac{1}{A} \int_{A} \bar{\alpha}_{S} d A \cong \frac{1}{A} \int_{A} \alpha_{S} d A
$$

A $0.2 \mathrm{~mm}$ of pipe wall roughness is adopted during simulation. Since the nature of wall-particle collisions influences the shear stress and turbulent energy flux at the walls, a specularity coefficient is defined for solid phase at the walls which quanti- 
fies the nature of these collisions. Its value varies between zero and unity. Unity corresponds to diffuse collisions which occur on a very rough wall whereas zero corresponds to specular collisions which occur on smooth frictionless walls. In the view of this, a value of 0.5 is selected which corresponds to wall quality between smooth frictionless walls and very rough walls. Apart this, no slip for liquid phase has been adopted at walls.

Pressure outlet boundary condition is adopted at the outlet boundary where mixture pressure is mentioned.

\section{Solution process and convergence criteria}

A CFD software 'FLUENT 6.3.26 has been used for simulating the flow using prescribed boundary conditions and turbulence model. A convergence criterion of $10^{-3}$ has been adopted for termination of iteration. To ensure satisfactory accuracy, stability and convergence of iterative process, a second order upwind discretization for momentum equation and first upwind discretization for volume fraction, turbulent kinetic energy and its dissipation are adopted.

\section{ANALYSIS OF RESULTS}

Simulation results are captured at the pipe outlet which is at $50 D$ length and also over the pipe length as applicable. The flow becomes fully developed much before reaching the pipe outlet. It is shown by plotting pressure gradient along the pipe length for particle size of $0.165 \mathrm{~mm}$. This supports the choice of location for obtaining simulation results. The simulation data for concentration distribution, velocity distributions, granular pressure, turbulent viscosity, turbulent kinetic energy and its
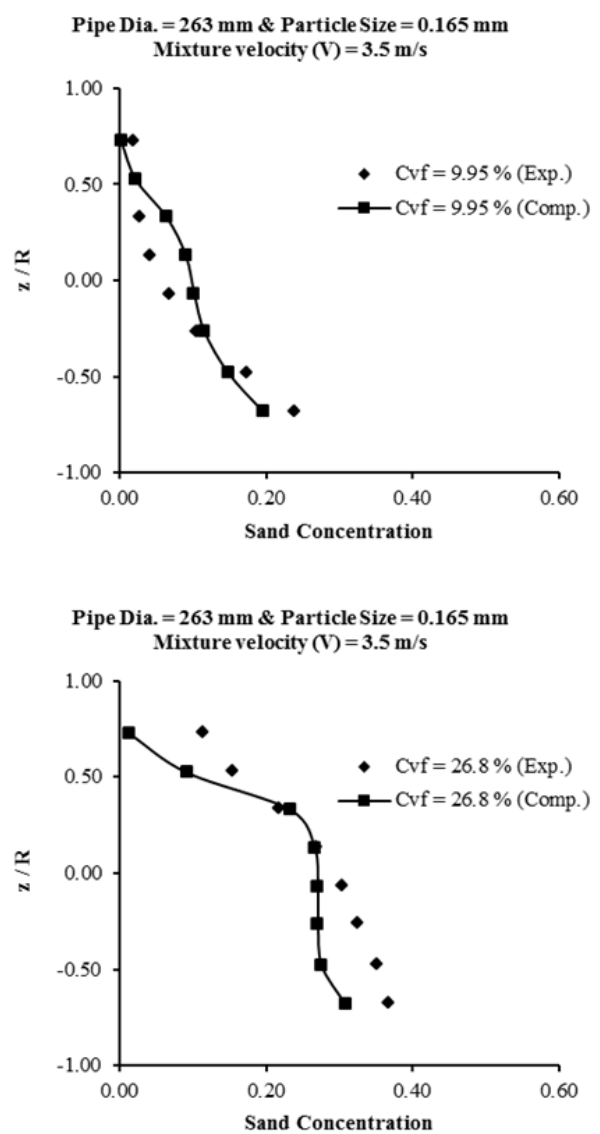

dissipation are observed at the pipe outlet whereas pressure gradient and friction factor are calculated over the whole length of the pipeline.

\section{Concentration profiles}

Figures 2-4 show comparison of experimental data of local volumetric concentration of solid with its simulated values along vertical centerline of the cross-section at pipe outlet Here $C_{v f}$ is the local volumetric concentration of solid calculated as the average value over the chord length (in y-direction) mathematically presented as shown below:

$C_{v f}^{\prime}=\frac{1}{2 y} \int_{-y}^{+y} \alpha_{s}\left(y, \frac{z}{R}\right) d y$

$z$ is the height from the centre of the pipe outlet which is at $(50 D, 0,0)$; and $R$ is the radius of the pipe. Here, radius, $R$, is preferred over diameter, $D$, as the experimental results were presented with respect to $z / R$ only.

Simulated results are in good agreement with corresponding experimental data for all three particles sizes viz. $0.165 \mathrm{~mm}$, $0.29 \mathrm{~mm}$ and $0.55 \mathrm{~mm}$. However, small deviations can be observed between these values for particle sizes $0.29 \mathrm{~mm}$ and 0.55 $\mathrm{mm}$. For particle size of $0.29 \mathrm{~mm}$, these deviations are observed for high efflux concentration cases (34\%) which are limited to upper half of the cross-section at both mixture velocities $(\mathrm{V}=4$ $\mathrm{m} / \mathrm{s} \& 4.7 \mathrm{~m} / \mathrm{s}$ ) under consideration. And, for particle size of $0.55 \mathrm{~mm}$, deviations are observed for all efflux concentrations under study for mixture velocity $3.9 \mathrm{~m} / \mathrm{s}$. However, with increase in mixture velocity $(4.4 \mathrm{~m} / \mathrm{s})$, these deviations get limited to high efflux concentration case only (30\%).
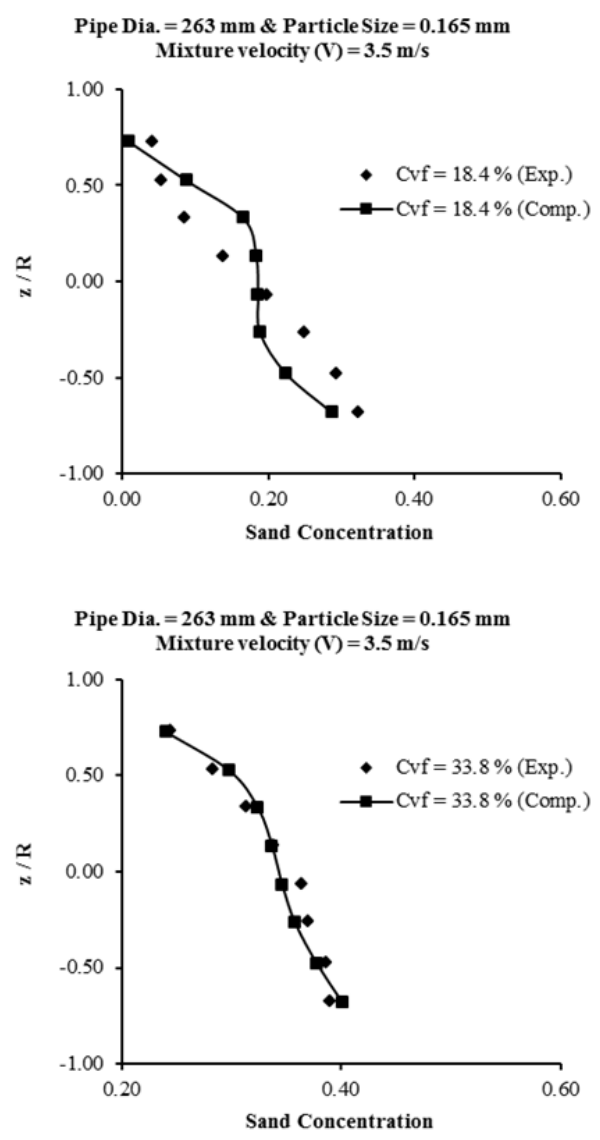

Fig. 2. Comparison of simulated and measured values of local volumetric sand concentration across vertical centre line of pipe outlet for particle size of $0.165 \mathrm{~mm}$ at different mixture velocities and efflux concentrations. 

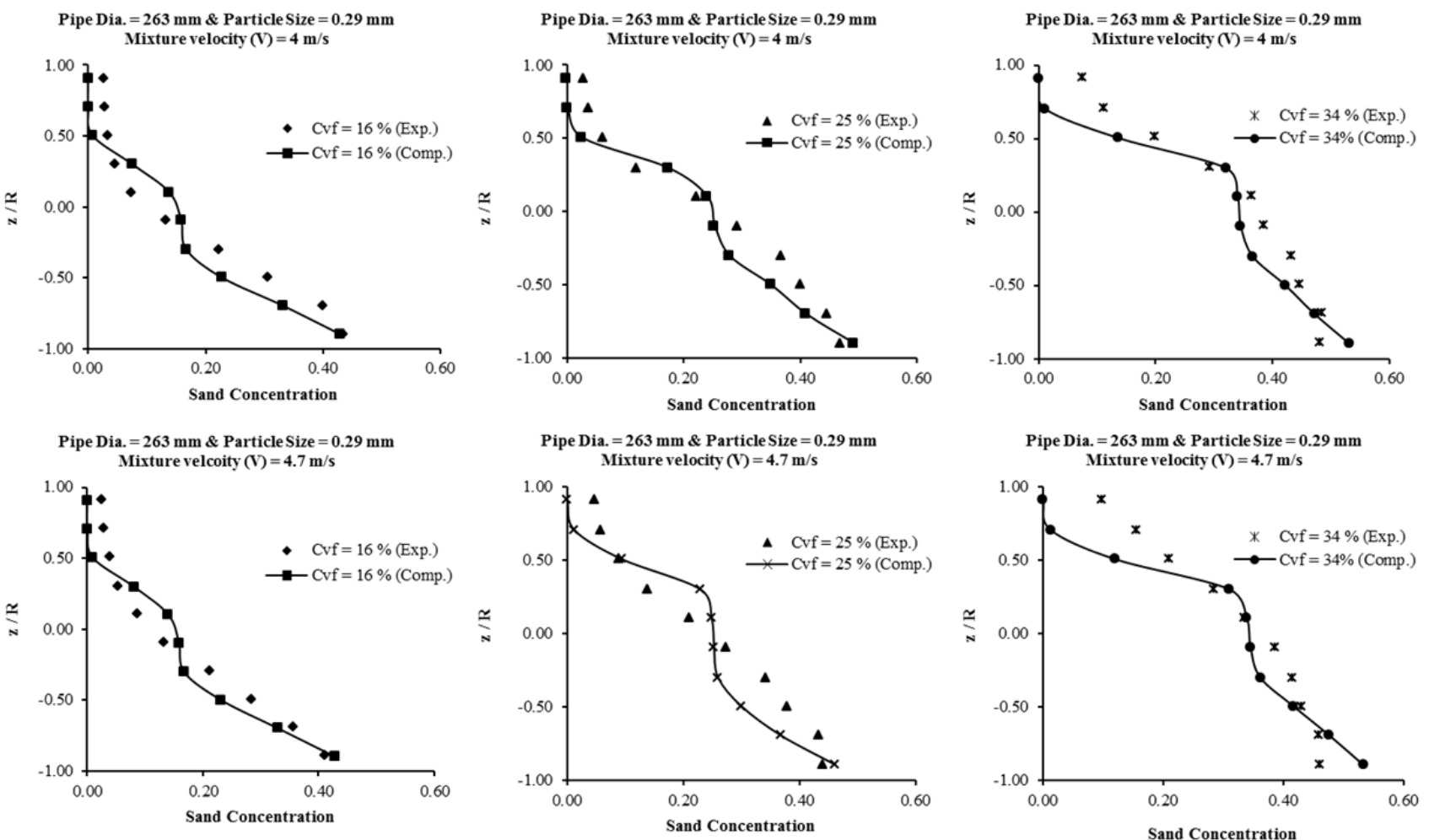

Fig. 3. Comparison of simulated and measured values of local volumetric sand concentration across vertical centre line of pipe outlet for particle size of $0.29 \mathrm{~mm}$ at different mixture velocities and efflux concentrations.
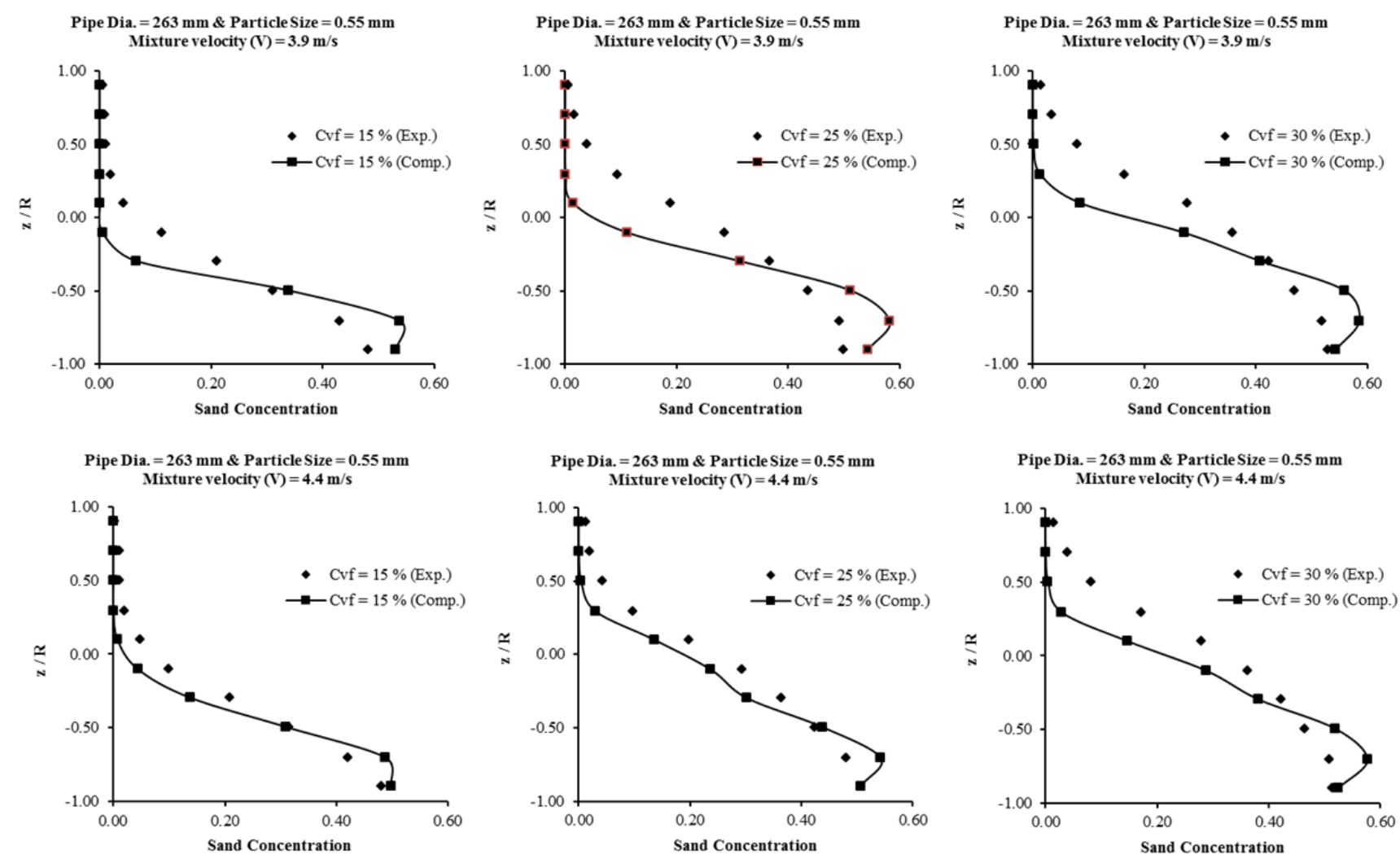

Fig. 4. Comparison of simulated and measured values of local volumetric sand concentration across vertical centre line of pipe outlet for particle size of $0.55 \mathrm{~mm}$ at different mixture velocities and efflux concentrations.

The possible reason for these deviations could be the abrasive rounding of solid particles by repeated passages during experiment, resulting generation of significant amount of fines distributed uniformly within the pipe. This is predominant in slurry flows with large size particles leading to possible in- crease in carrier density. Since information of this aspect was not reported in the published work showing experimental data, the same is not incorporated during simulations leading to possible deviations in the output data as compared to corresponding experimental results. 


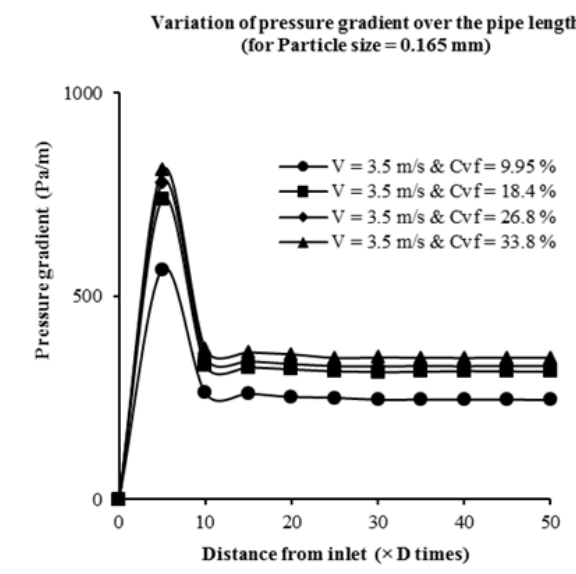

(a)

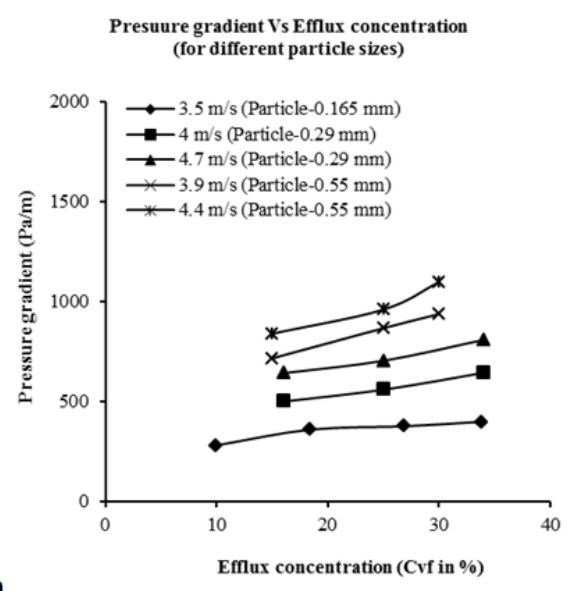

(b)

Fig. 5. (a) Variation of pressure gradient over the pipe length for particle size of $0.165 \mathrm{~mm}$ and mixture velocity of $3.5 \mathrm{~m} / \mathrm{s}$ at different efflux concentrations. (b) Simulation results of average pressure gradient for all three particle sizes at different mixture velocities and efflux concentrations.

\section{Pressure gradient}

Pressure gradient (in $\mathrm{Pa} / \mathrm{m}$ ) is one of the important inputs required for the designing of slurry pipe line. Its variation over the pipe length is plotted in Figure 5 (a) for particle size of $0.165 \mathrm{~mm}$ to ascertain the downstream length for flow development. It is found that pressure gradient becomes almost constant after 25-30D downstream length for all cases. This supports the selection of pipe outlet, which is $50 D$ downstream, as the plane for capturing data of various flow parameters presented in the paper.

Figure 5 (b) shows variation of average pressure gradient (in $\mathrm{Pa} / \mathrm{m})$ with efflux concentration $\left(C_{v f}\right)$ for different particle sizes at various mixture velocities. Pressure gradient is found to be increasing with increase in efflux concentration for all particle sizes at all mixture velocities. And its rate increases either with particle size for a given velocity or with mixture velocity for a given particle size.

Similar trends have also been reported in many past researches such as Kumar et al. (2003), Kaushal et al. (2005), Kaushal et al. (2012) etc.; but with different pipe \& particle sizes having different sets of fluid \& flow characteristics.

\section{Velocity distribution}

Figure 6 shows distribution of non-dimensional velocity of solid phase across vertical centerline at pipe outlet for all three particle sizes with different mixture velocities. The velocity of solid phase $\left(V_{s}\right)$ is non-dimensionalized using mixture velocity at pipe inlet.

For particle size of $0.165 \mathrm{~mm}$, the distribution is found to be parabolic for lower value of efflux concentration which becomes progressively flatter with efflux concentration. At higher particle sizes i.e. $0.29 \mathrm{~mm} \& 0.55 \mathrm{~mm}$, the distribution becomes asymmetric due to shifting of area of higher velocity in the lower half of the pipe cross-section. This shift is found to be more at higher efflux concentrations for a given particle size. However, for a given efflux concentration, the shift is more for flows with bigger particle.

The asymmetric nature of distribution of non-dimensional velocity of solid phase across vertical centerline at pipe outlet for bigger particles can be attributed to the heaviness of particle supported by the carrier.

Many previous researches also highlighted similar trends for solid velocity distribution presented in different manner. Nota- ble among it are Ling et al. (2003), Lin and Ebadian (2008), Kaushal et al. (2012) etc.

\section{Growth of friction factor}

Figure 7 shows the growth of friction factor $(f)$ over the length of pipe for all three particle sizes under consideration at different mixture velocities and efflux concentrations. Friction factor is calculated using weighted friction factor of each phase. It is given as below:

$$
f_{\text {mixture }}=\left(1-C_{v f}\right) \times f_{\text {phase I }}+C_{v f} \times f_{\text {phase II }}
$$

Friction factor attains a nearly constant value soon after entering the pipeline for all mixture velocities and particle sizes. Its value for a particular particle size and mixture velocity decreases marginally with increase in efflux concentration of flow throughout the pipe length. Also, for a particular particle size, its value increases with mixture velocity. Also, this can be attributed to formation of bed type layers moving smoothly over each other when the flow efflux concentration is higher.

Researches such as Ling et al. (2003), Lin and Ebadian (2008) etc. also supported the trends of friction factor reported above.

\section{Granular pressure}

Granular pressure, also called collisional particle pressure, is the force per unit area exerted by a moving granular medium on the pipe walls carrying the mixture. It is a measure of the momentum transfer due to streaming particles. The reaction to this on primary medium which in present case is water results in pressure drop.

Granular pressure is calculated using the kinetic energy of fluctuating particle motion and particle collisions. It is present in the form of solid pressure gradient in momentum equation of solid phase.

Figure 8 shows variation of granular pressure (in $\mathrm{Pa}$ ) with efflux concentration for all three particle sizes. Its value increases with increase in efflux concentration for all particle sizes and mixture velocity due to enhanced momentum transfer between particles and the walls. It is also noticed that the values of granular pressure for particle size of $0.55 \mathrm{~mm}$ increases significantly as compared to smaller particle sizes. It may be attributed to increased momentum exchange and kinetic energy 

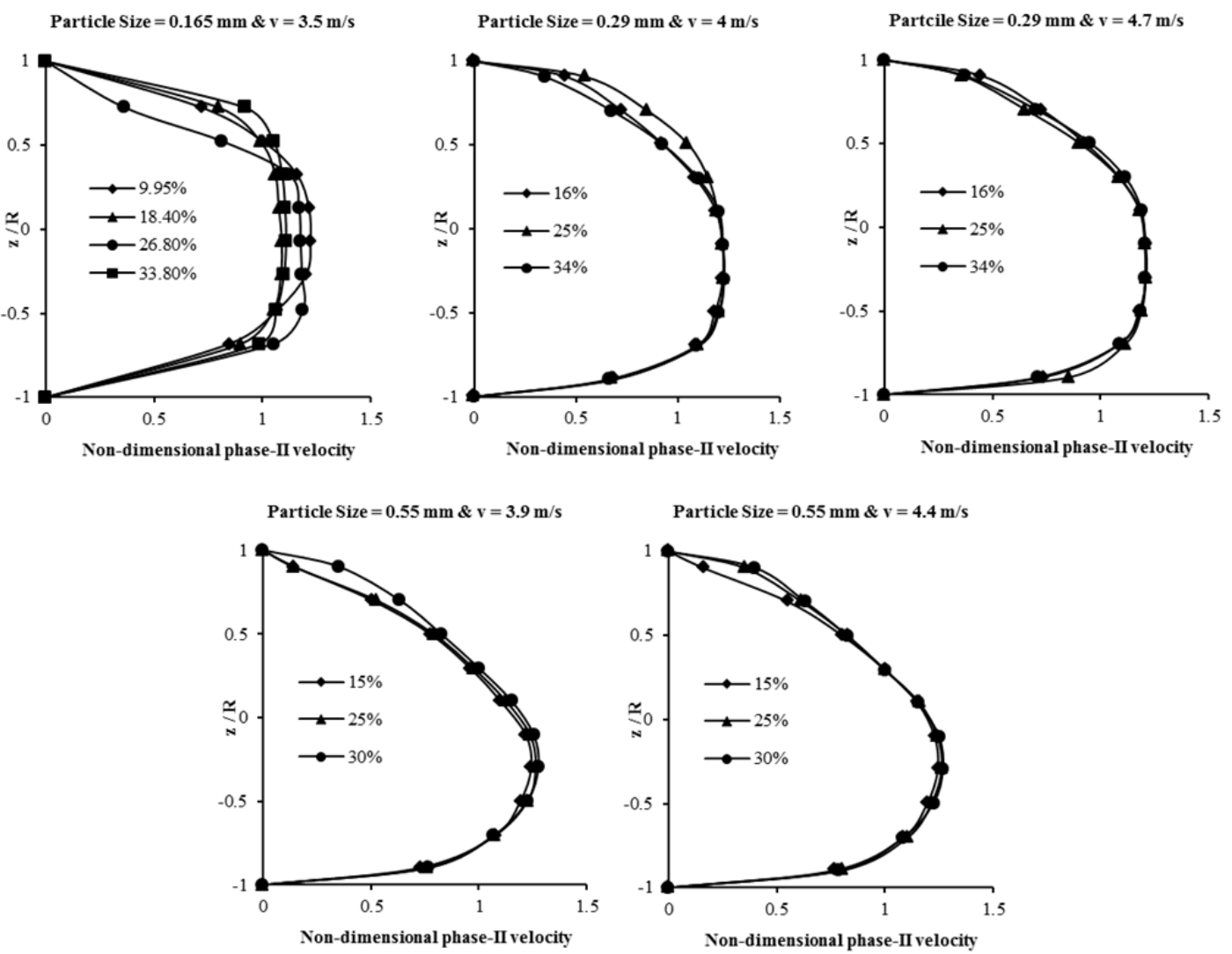

Fig. 6. Simulation results of local sand (phase-II) velocity across vertical centre line at pipe outlet for all three particle sizes at different mixture velocities and efflux concentrations.
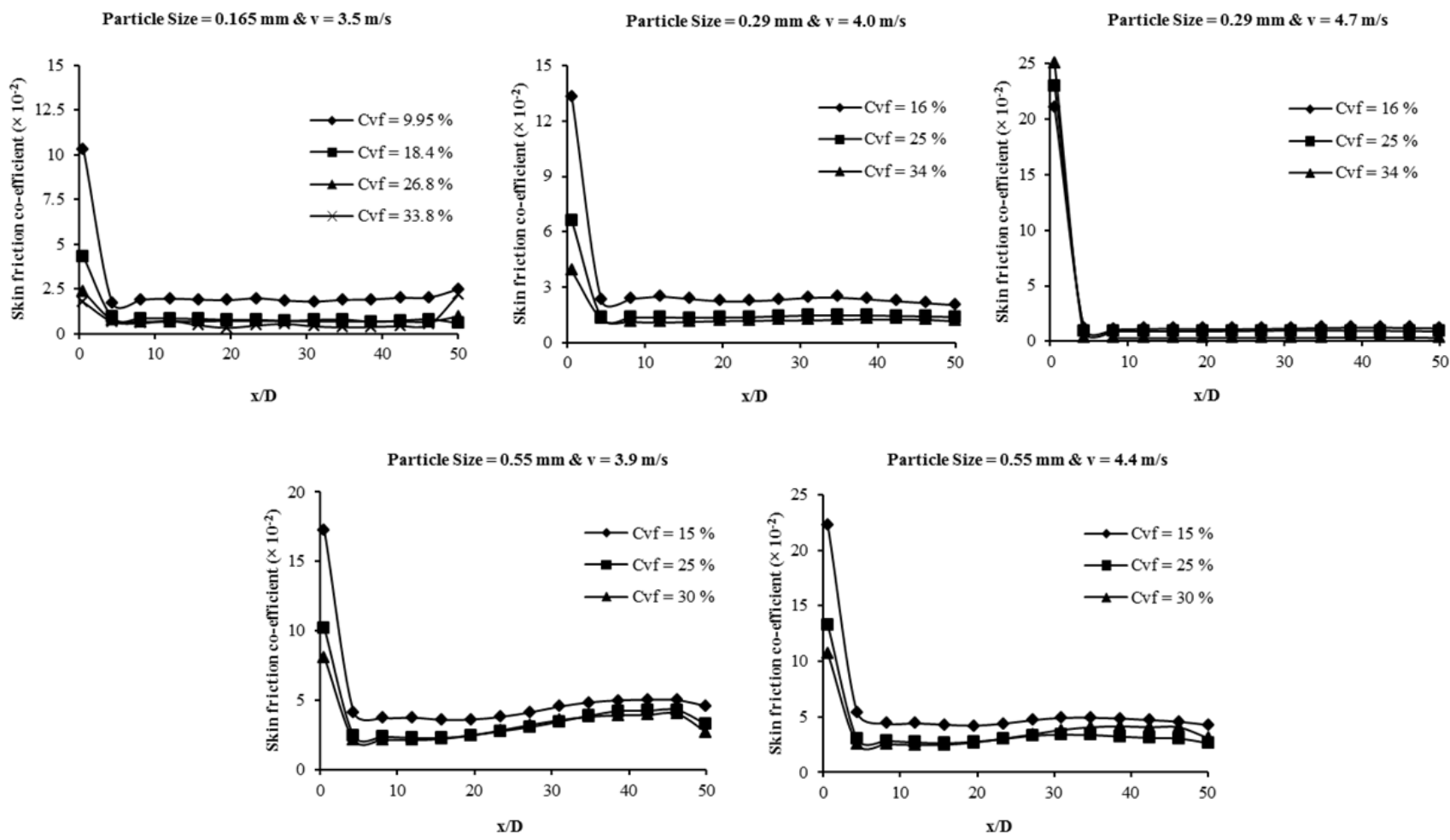

Fig. 7. Simulation results showing growth of friction factor along pipe length for all three particle sizes at different mixture velocities and efflux concentrations.

of fluctuation particles due to bigger grain. Also, its value decreases with increase in mixture velocity for all efflux concentration cases due to increased turbulence at higher mixture velocity at this grain size leading to loss of energy. The same in case of $0.29 \mathrm{~mm}$ particle size is found to be negligible. 


\section{Turbulent viscosity}

Modeling of turbulent viscosity of the slurry during the flow is one of the most critical aspects of the slurry flows. It depends upon the interaction between particles and between particles and the fluid. Due accounting for these effects in detail is still a challenging problem for the researchers.

Figure 9 shows variation of turbulent viscosity of slurry flow with efflux concentration for all three particle sizes at different mixture velocities. Turbulent viscosity of the slurry flow is found to be decreasing with increase in efflux concentration for all particle sizes. And, the same at any particular particle size decreases with increase in mixture velocity. Drop in the value of turbulent viscosity of slurry flows with mixture velocity is found to be more for flows with bigger particle sizes. Variation in turbulent viscosity with efflux concentration, mixture velocity or with particle size are in accordance to the variation in turbulence characteristics of the flow described in subsequent paragraphs.
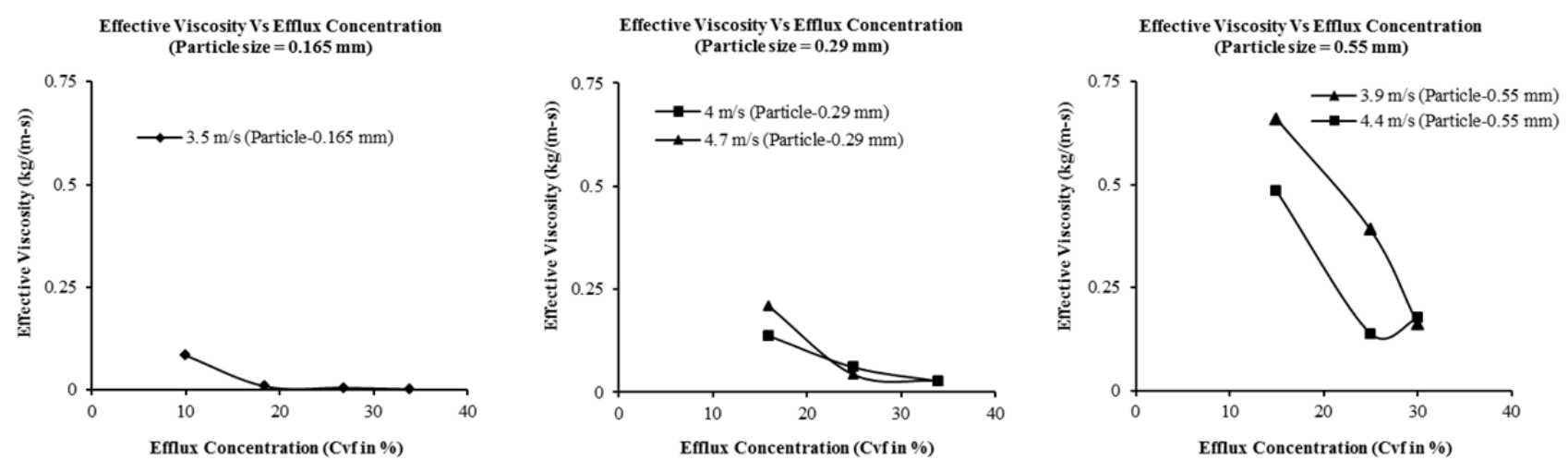

Fig. 9. Simulation results of variation in turbulent viscosity at pipe outlet with efflux concentration for all three particle sizes at different mixture velocities.
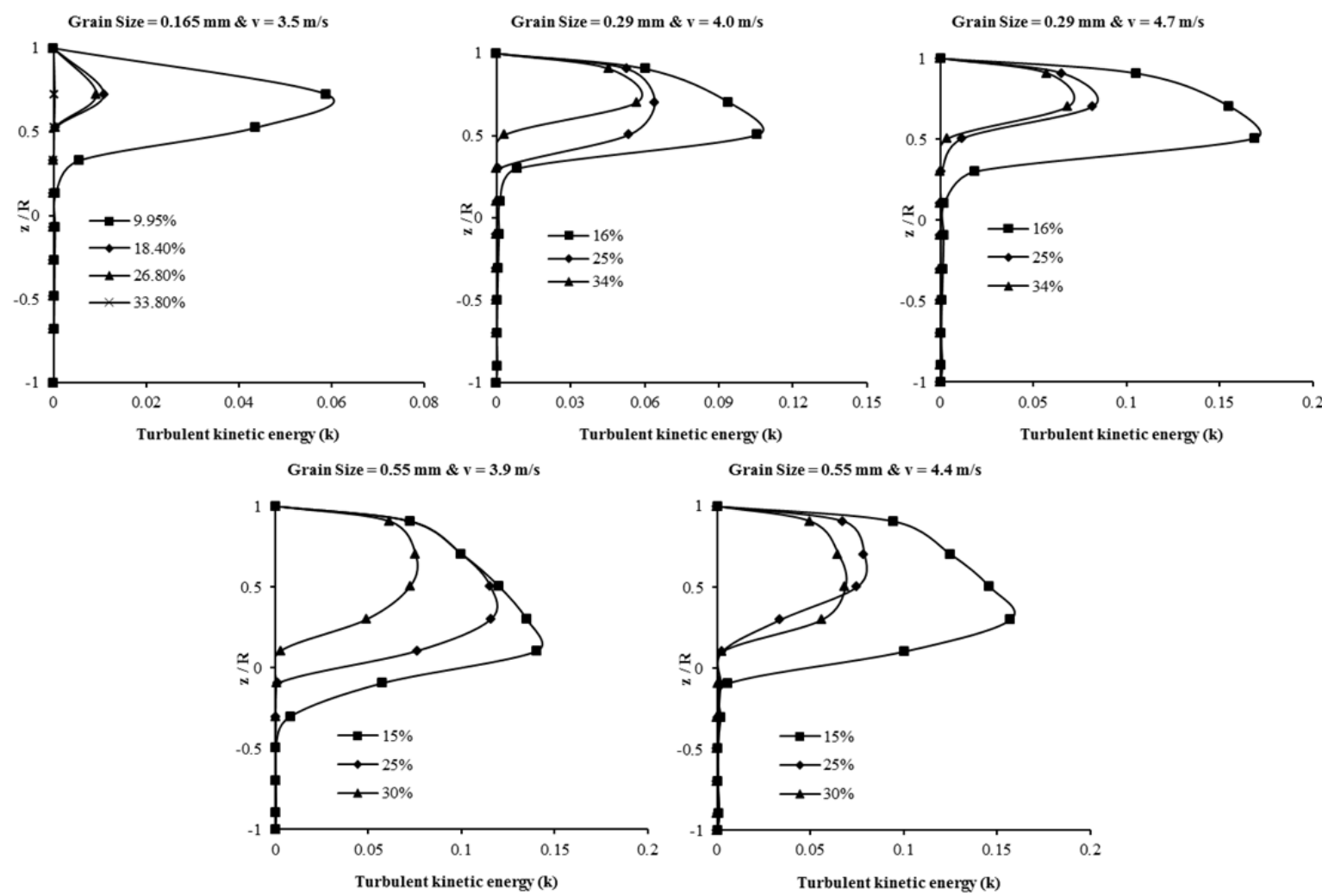

Fig. 10. Simulation results of turbulent kinetic energy across vertical centre line of pipe outlet for all three particle sizes at different mixture velocities and efflux concentrations. 


\section{Turbulent kinetic energy $\&$ its dissipation}

Distribution of turbulent kinetic energy $(k)$ of primary phase at any cross-section shows the turbulence level across it. Figure 10 shows distribution of turbulent kinetic energy of primary phase across vertical centerline at pipe outlet for all three particle sizes with different mixture velocities and efflux concentrations. Region of higher turbulence is found to be located in the upper half of the pipe outlet cross-section. This is justified also as it is the region where secondary phase is loosely located resulting more turbulence.

It is also observed that the spread of the region of higher turbulence located in the upper half of the pipe outlet cross-section reduces with increase in efflux concentration for all particle sizes and mixture velocities. This is because increase in efflux concentration results in movement of more particles near the core region and in the upper half leading to decreased turbulence level.

Also, for a given particle size, spread of the region of higher turbulence is found to be marginally decreasing with increase in mixture velocity. This can also be attributed to the increased particle movement in upper half of the pipe outlet cross-section at higher mixture velocities.

Dissipation of turbulent kinetic energy $(\varepsilon)$ represents the reduction of turbulence with time which is one of the important parameters in the overall understanding of turbulence in turbulent flows. Figure 11 shows distribution of dissipation of turbulent kinetic energy across vertical centerline at pipe outlet for all three particle sizes with different mixture velocities and efflux concentrations. The distribution of turbulent kinetic energy \& its dissipation are found to be quite similar as expected. This is so because dissipation of anything must follow the path of its growth \& development.

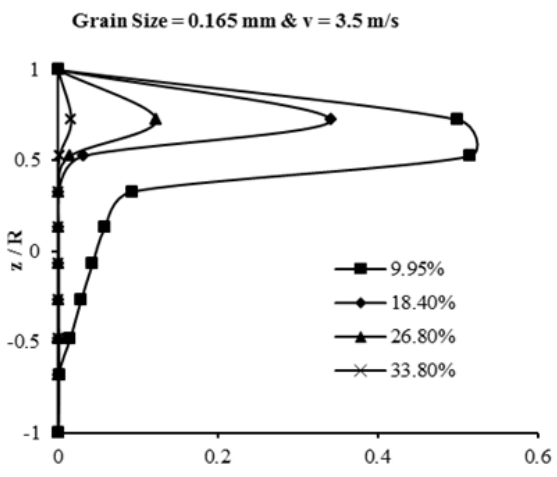

Turbulent kinetic energy disipation $(\varepsilon)$

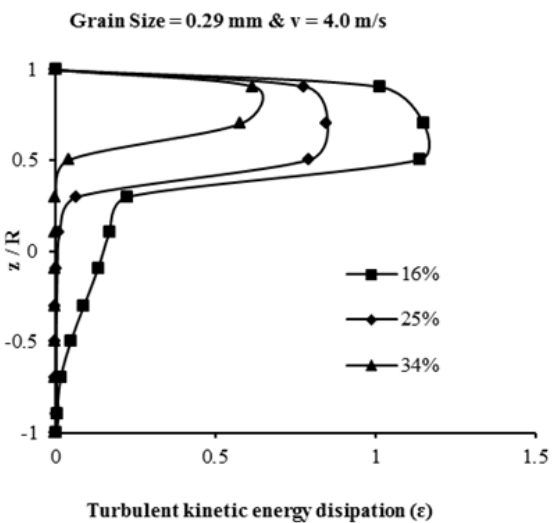

Turbulent kinetic energy disipation $(\varepsilon)$

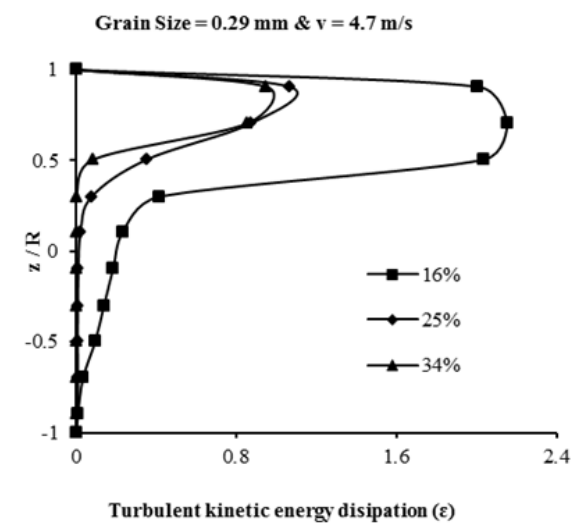

Turbulent kinetic energy disipation $(\varepsilon)$
Grain Size $=0.55 \mathrm{~mm} \mathrm{\&} \mathrm{v}=3.9 \mathrm{~m} / \mathrm{s}$

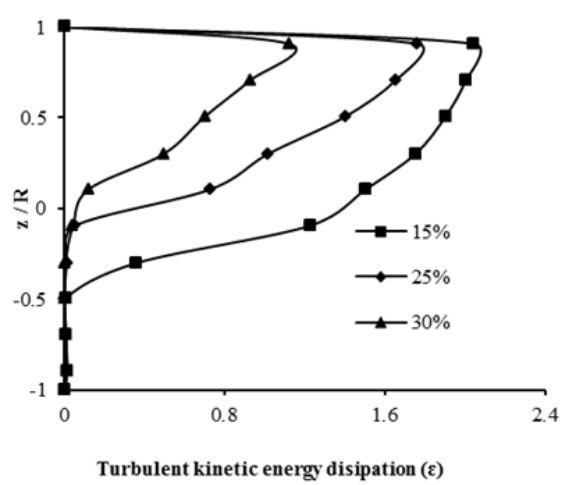

Grain Size $=0.55 \mathrm{~mm} \& \mathrm{v}=4.4 \mathrm{~m} / \mathrm{s}$

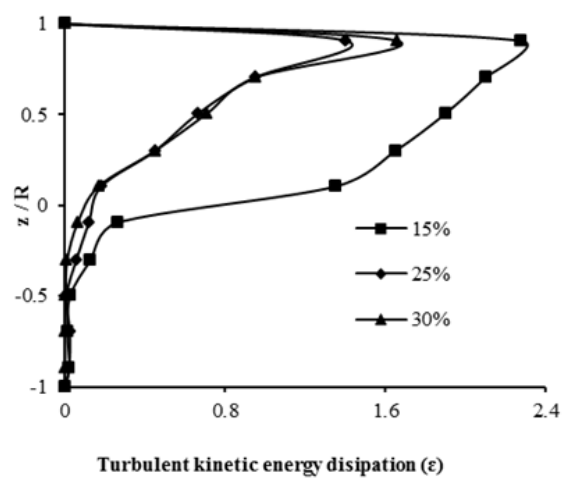

Fig. 11. Simulation results of dissipation of turbulent kinetic energy across vertical centre line of pipe outlet for all three particle sizes at different mixture velocities and efflux concentrations.

\section{CONCLUSIONS}

The paper presents three-dimensional CFD analysis of twophase (sand-water) slurry flows through $263 \mathrm{~mm}$ diameter pipe in horizontal orientation for mixture velocity range of 3.5-4.7 $\mathrm{m} / \mathrm{s}$ and efflux concentration range of $9.95-34 \%$ with three particle sizes viz. $0.165 \mathrm{~mm}, 0.29 \mathrm{~mm}$ and $0.55 \mathrm{~mm}$ with density $2650 \mathrm{~kg} / \mathrm{m}^{3}$. Local volumetric concentration of solid phase across pipe outlet is very well simulated for all three particles sizes under consideration. It is observed that pressure gradient has a great dependency on efflux concentration for all particle sizes at all mixture velocities. A symmetric solid phase velocity distribution is obtained for fine particles which becomes progressively asymmetric with courser particles. Friction factor growth achieves a constant value at small distance downstream the inlet. Trends remain unchanged for all mixture velocities and particle sizes. It can very well be concluded from this study that momentum transfer between particles and the walls, represented by granular pressure, gets hugely effected by efflux concentration and particle size. Also, turbulent viscosity of the slurry flow shows decline with increase in efflux concentration for all particle sizes.

Study of flow turbulence is the highlight of this paper as not many researchers have presented this aspect of slurry flows. It is observed during this study that turbulence dominates in the region of lesser solid concentration. Also, its spread reduces with increase in efflux concentration for all particle sizes and mixture velocities. 
This paper helps in improving the understanding of twophase slurry flows with different particle sizes. However, scatter in data of various flow parameters especially in case of slurry flows with bigger particle sizes indicates that the model used in the present study needs further development. Also, the choice of coefficient of lift, coefficient of drag, restitution coefficient and wall boundary conditions needs to be further supported by thorough parametric studies.

\section{REFERENCES}

Antaya, C.L., Adane, K.F.K., Sanders, R.S., 2012. Modeling Concentrated Slurry Pipeline Flows.Proc. ASME. 44755, Volume 1: Symposia, Parts A and B 1659.

Aude, T.C., Thomson, T.L., Wasp, E.J., 1974. Economics of slurry pipeline systems. In: Proc. Hydro transport 3. BHRA Fluid Engineering, Cranfield, Bedford, England, paper K2.

Aude, T.C., Thompson, T.L., Wasp, E.J., 1975. Slurry pipeline system. Oil and Gas Journal, pp.66.

Chen, L., Duan, Y., Pu, W., Zhao, C., 2009. CFD simulation of coal-water slurry flowing in horizontal pipelines. Korean Journal Chemical Engineering, 26, 4, 1144-1154.

Doron, P., Granica, D., Barnea, D., 1987. Slurry flow in horizontal pipes-experimental and modeling. International Journal of Multiphase Flow, 13, 535-547.

Ekambara, K., Sanders, R.S., Nandakumar, K., Masliyah, J.H., 2009. Hydrodynamic simulation of horizontal slurry pipeline flow using ANSYS-CFX. Ind. Eng. Chem. Res., 48, 81598171.

Ghanta, K.C., Purohit, N.K., 1999. Pressure drop prediction in hydraulic transport of bi-dispersed particles of coal and copper ore in pipeline. The Canadian Journal of Chemical Engineering, 77, 127-131.

Gidaspow, D., Bezburuah, R., Ding, J., 1992. Hydrodynamics of circulating fluidized beds, kinetic theory approach in fluidization. In: Proceedings of the 7th Engineering Foundation Conference on Fluidization, pp. 75-82.

Gillies, R.G., Shook, C.A., Wilson, K.C., 1991. An improved two layer model for horizontal slurry pipeline flow. The Canadian Journal of Chemical Engineering, 69, 173-178.

Gillies R.G., Shook, C.A., 1994. Concentration Distributions of sand slurries in horizontal pipe flow. Particulate Science and Technology: An International Journal, 12, 1, 45-69.

Gillies, R.G., Hill, K.B., Mckibben, M.J., Shook, C.A., 1999. Solids transport by laminar Newtonian flows. Powder Technology, 104, 269-277.

Gillies, R.G., Shook, C.A., 2000. Modeling high concentration settling slurry flows. The Canadian Journal of Chemical Engineering, 78, 709-716.

Gopaliya, M.K., Kaushal, D.R., 2015. Analysis of effect of particle size on various parameters of slurry flow through pipeline using CFD. Particulate Science and Technology: An International Journal, 33, 4, 369-384.

Karabelas, A.J., 1977. Vertical distribution of dilute suspensions in turbulent pipe flow. AIChE Journal, 23, 426-434.

Kaushal, D.R., Tomita, Y., 2002. Solid concentration profiles and pressure drop in pipeline flow of multisized particulate slurries. International Journal of Multiphase Flow, 28, 1697-1717.

Kaushal, D.R., Tomita, Y., 2003. Comparative study of pressure drop in multisized particulate slurry flow through pipe and rectangular duct. International Journal of Multiphase Flow, 29, 1473-1487.

Kaushal, D.R., Sato, K., Toyota, T., Funatsu, K., Tomita, Y., 2005. Effect of particle size distribution on pressure drop and concentration profile in pipeline flow of highly concen- trated slurry. International Journal of Multiphase Flow, 31, 809-823.

Kaushal, D.R., Thinglas, T., Tomita, Y., Kuchii, S., Tsukamoto, H., 2012. CFD modeling for pipeline flow of fine particles at high concentration. International Journal of Multiphase Flow, 43, 85-100.

Krampa-Morlu, F.N., Bergstrom, D.J., Bugg, J.D., Sanders, R.S., Schaan, J., 2004. Numerical simulation of dense coarse particle slurry flows in a vertical pipe. In: 5th International Conference on Multiphase flow, ICMF'04, Yokohama, Japan.

Kumar, U., Mishra, R., Singh, S., Seshadri, V., 2003. Effect of particle gradation on flow characteristics of ash disposal pipelines. Powder Technology, 132, 39-51.

Launder, B.E., Spalding, D.B., 1974. The numerical computation of turbulent flows. Computational Methods in Applied Mechanics \& Engineering, 3, 269-289.

Lin, C.X., Ebadian, M.A., 2008. A numerical study of developing slurry flow in the entrance region of a horizontal pipe. Computers \& Fluids, 37, 965-974.

Ling, J., Skudarnov, P.V., Lin, C.X., Ebadian, M.A., 2003. Numerical investigations of liquid-solid slurry flows in a fully developed turbulent flow region. International Journal of Heat and Fluid Flow, 24, 389-398.

Lun, C.K.K., Savage, S.B., Jeffrey, D.J., Chepurniy, N., 1984. Kinetic theories for granular flow: inelastic particles in couette flow and slightly inelastic particles in a general flow field. Journal of Fluid Mechanic, 140, 223-256.

Ma, L., Huang, C., Xie, Y., Jiang, J., Tufa, K.Y., Hui, R., 2015. Modeling of erodent particle trajectories in slurry flow. Wear, 334-335, 49-55.

Mishra, R., Singh, S.N., Seshadri, V., 1998. Improved model for prediction of pressure drop and velocity field in multisized particulate slurry flow through horizontal pipes. Powder Handling and Processing Journal, 10, 279-289.

O'Brien, M.P., 1933. Review of the theory of turbulent flow and its relations to sediment transport. Transaction of the American Geophysical Union 14, 487-491.

Roco, M.C., Shook, C.A., 1983. Modeling of slurry flow: The effect of particle size. The Canadian Journal of Chemical Engineering, 61, 494-503.

Roco, M.C., Shook, C.A., 1984. Computational methods for coal slurry pipeline with heterogeneous size distribution. Powder Technology, 39, 159-176.

Rouse, H., 1937. Modern conceptions of the mechanics of fluid turbulence. Transactions of ASCE, 102, 463-505.

Seshadri, V., 1979. Basic process design. In: Proc. of Workshop on Hydraulic transportation of mineral ores, IIT, Delhi.

Seshadri, V., 1982. Basic process design for a slurry pipeline. In: Proc. The Short Term Course on Design of pipelines for transporting liquid and solid materials, IIT, Delhi.

Seshadri, V., Malhotra, R.C., Sundar, K.S., 1982. Concentration and size distribution of solids in a slurry pipeline. In: Proc. 11th Nat. Conference on Fluid mechanics and fluid power, B.H.E.L., Hyderabad.

Shook, C.A., Daniel, S.M., 1965. Flow of suspensions of solids in pipeline: I. Flow with a stable stationary deposit. The Canadian Journal of Chemical Engineering, 43, 56-72.

Shook, C.A., Daniel, S.M., Scott, J.A., Holgate, J.P., 1968. Flow of suspensions in pipelines. The Canadian Journal of Chemical Engineering, 46, 238-244.

Sundqvist, A., Sellgren, A., Addie, G., 1996. Slurry pipeline friction losses for coarse and high density products. Powder Technology, 89, 19-28. 
Wang, J., Zhang, T., Wang, S., 2013a. Heterogeneous ice slurry flow and concentration distribution in horizontal pipes. International Journal of Heat and Fluid Flow, 44, 425-434.

Wang, J., Wang, S., Zhang, T., Liang, Y., 2013b. Numerical investigation of ice slurry isothermal flow in various pipes. International Journal of Refrigeration, 36, 70-80.

Wasp, E.J., Aude, T.C., Kenny, J.P., Seiter, R.H., Jacques, R.B., 1970. Deposition velocities, transition velocities and spatial distribution of solids in slurry pipelines. In: Proc. Hydrotransport 1, BHRA Fluid Engineering, Coventry, UK, paper H4.2, pp. 53-76.
Wilson, K.C., Clift, R., Sellgren, A., 2002. Operating points for pipelines carrying concentrated heterogeneous slurries. Powder Technology, 123, 19-24.

Wu, D., Yang, B., Liu, Y., 2015. Pressure drop in loop pipe flow of fresh cemented coal gangue-fly ash slurry: Experimental and simulation. Advanced Powder Technology, 26, 920-927.

Received 25 January 2016 Accepted 6 May 2016 